\title{
Histological findings in direct inguinal hernia
}

\author{
Investigating the histological changes of the herniated groin \\ looking forward to ascertain the pathogenesis of hernia disease
}

\author{
G. Amato - A. Agrusa - G. Romano • \\ G. Salamone $\cdot$ G. Cocorullo $\cdot$ S. A. Mularo $\cdot$ \\ S. Marasa - G. Gulotta
}

Received: 27 October 2011 / Accepted: 11 December 2012/Published online: 4 January 2013

(C) Springer-Verlag France 2013

\begin{abstract}
Background The study is focused on recognizing the histological changes of the structures close to and around the hernia opening in patients having direct inguinal hernia. Methods In 15 patients with primary bilateral direct inguinal hernia who underwent a Stoppa open posterior inguinal hernia repair, tissue specimens from the abdominal wall surrounding a direct hernia border were excised for histological examination. These findings in patients with direct inguinal hernia were compared with tissue specimens excised from the fossa inguinalis media of cadavers without hernia.

Results Significant degenerative modifications such as fibrohyaline degeneration and fatty substitution of the muscle fibers were seen in the biopsy samples. Inflammatory infiltration with lympho-histiocitary elements, artery sub-occlusion and vascular congestion were also constantly identified. Noteworthy injuries of the nervous structures such as edema, degenerative fibrosis and atrophy were also detected. No comparable tissue damage was witnessed in the control samples.
\end{abstract}

\footnotetext{
G. Amato $(\bowtie) \cdot$ A. Agrusa - G. Romano - G. Salamone ·

G. Cocorullo · G. Gulotta

Department of General Surgery, Urgency and Organ

Transplantation, University of Palermo, Via Rapisardi 66, 90144 Palermo, Italy

e-mail: amatomed@gmail.com
}

\section{S. A. Mularo}

Department of Anesthesia and Intensive Care,

University of Palermo, Palermo, Italy

\section{S. Marasa}

Department of Pathological Anatomy and Histology,

University of Palermo, Palermo, Italy
Conclusion Presence of inflammatory infiltration, vascular damage and regressive nerve lesions, as well as fibrohyaline degeneration and fatty dystrophy of the muscle fibers are the features seen within the examined structures surrounding the direct hernia opening. These findings could represent a reason for a structural and functional weakening of the inguinal region. Consequently, the described results lead the authors to depict these changes as a plausible cause of direct inguinal hernia protrusion.

Keywords Inguinal hernia - Direct - Etiology · Inflammation - Muscles · Fibrosis · Hyaline . Muscular dystrophy $\cdot$ Nerve degeneration

\section{Introduction}

Inguinal hernia repair is one of the most frequent surgical procedures. The etiopathogenesis of inguinal hernia has been examined for over 100 years. However, despite the constant improvement in surgical techniques and materials used for inguinal hernia repair, few effective advances in hernia genesis have been reported. Studies examining collagen tissue changes related to hernia development do not seem having disclosed a full link to the pathogenesis of the disease [1-3]. A dysfunction in a sphincter-like motion of the internal inguinal ring was suggested as a potential failure mechanism causing inguinal hernia [4]. Furthermore, a massive fibrohyaline degeneration of the muscle fibers, inflammatory infiltration and vascular congestion within the muscular frame of the internal inguinal ring were reported in patients with indirect inguinal hernia [5]. This structural damage is a possible factor underlying the impairment of the defensive closure mechanism of the internal ring, thus allowing the protrusion of indirect 
inguinal hernia through the inguinal canal. The aim of the present study was to examine whether equivalent histological changes are present in the tissues surrounding the hernia opening in subjects with direct inguinal hernia.

\section{Materials and methods}

This study was approved by the Ethics Committee of the University Hospital of Palermo. In 15 consented patients who underwent open posterior hernia repair, according to Stoppa, biopsies were taken for histological study from the abdominal wall structures neighboring the hernia openings (30 hernias in total). Patients had a mean age of 61 years (range 39-75 years). All patients had bilateral primary direct inguinal hernia. We developed a standardized biopsy technique to obtain biopsies that could be compared between subjects with and without direct inguinal hernia. This method involved using the hernia defect as the landmark and then bisecting the hernia defect with a vertical line. The central point of the lower border was used as the starting point. From here, a line was drawn out at $45^{\prime}$ to the right of vertical, until the upper border of the defect was reached. Distances along this line of 1 and $2 \mathrm{~cm}$ distal to the border were measured, and a full thickness biopsy of $0.5 \times 0.5 \mathrm{~cm}$ was taken at each point (Fig. 1a).

The procedure was repeated at $45^{\prime}$ to the left of the vertical, and biopsies were collected at 1 and $2 \mathrm{~cm}$ as above. As controls tissue specimens with the same dimensions were excised from the inguinal area from 15 autopsied male cadavers without hernia. The control cadavers had an average age of 63 years (range $42-77$ years). The inguinal ligament was used as a key landmark for collection of biopsies. Parallel to this ligament and above we drew three lines at a distance of $0.5,1.5$ and $2.5 \mathrm{~cm}$. The tissue specimens were excised every $0.5 \mathrm{~cm}$ along these three lines, starting at $0.5 \mathrm{~cm}$ medial from the epigastric vessels until $0.5 \mathrm{~cm}$ from the lateral border of the rectus muscle (Fig. 1b). Using this method, we achieved an acceptable histological mapping of the entire fossa inguinalis media. Although this was not a perfect solution, and despite that our study design was not blinded to the pathologists, we felt that this repeatable measurement method of direct hernia versus controls specimens gave us a comparable tissue samples from the same anatomical areas. In the control cadavers, all tissue specimens were biopsied within 24-48 h of death. All samples were subject to histological study. To prevent modification of the excised tissue specimen, immediately after tissue excision the specimens were fixed in $10 \%$ neutral buffered formalin for at least $12 \mathrm{~h}$. After routine tissue processing, sections were cut at 4-6 mm and stained with Masson, PAS, Azan-Mallory, NSE, CD 20 and Hematoxylin eosin.

\section{Results}

Histological examination of the tissue excised from the hernia patients demonstrated numerous significant histological alterations. In all biopsy specimens, there was clear evidence of diffuse hyaline and fibrotic degeneration of the muscle fibers (Figs. 2, 3, 4). In addition, the muscular structures affected by fibrohyaline degeneration were surrounded by evident fibroadipous substitution of the muscle
Fig. 1 Excision site of the biopsy samples. a In living patients with direct hernia, a yellow line connects the lowest and the highest point of the hernia opening. Starting from the lowest point a $45^{\circ}$ inclined line transverses the figure on the right and on the left. 5 excisions (times symbol) are made: $1 \mathrm{~cm}$ above and $2 \mathrm{~cm}$ above the junction of both $45^{\circ}$ angled lines. b Biopsy samples (times symbol) in control cadavers without hernia were excised from the Hesselbach's triangle every $0.5 \mathrm{~cm}$ along three lines, starting $0.5 \mathrm{~cm}$ medial from the epigastric vessels until $0.5 \mathrm{~cm}$ from the lateral border of the rectus muscle
A

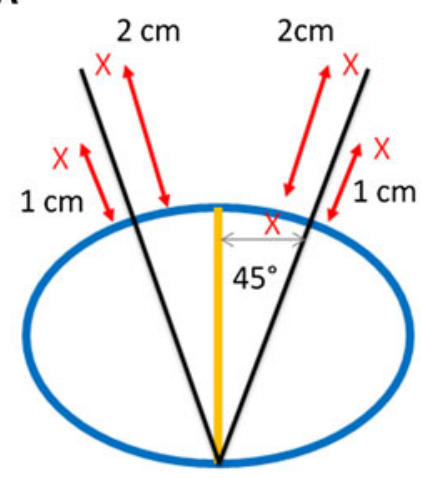

Hernia defect and excision sites in living patients

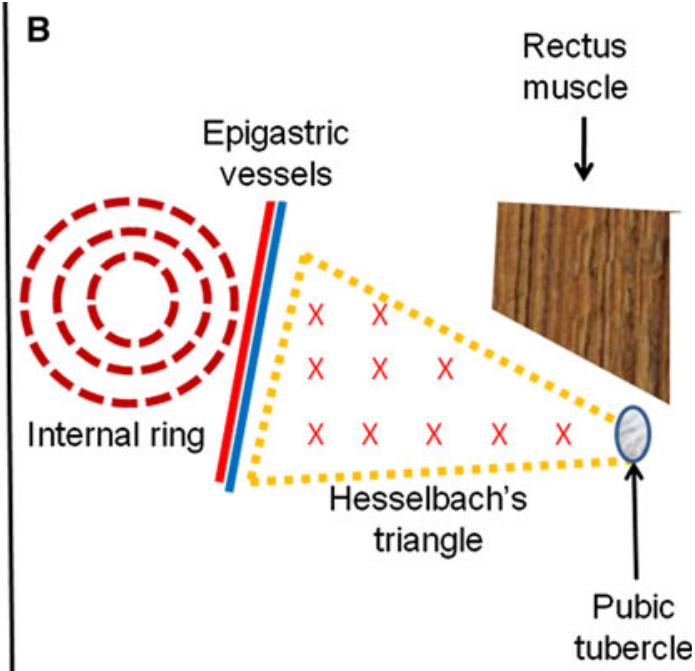

Biopsy places in control cadavers 
fibers (Figs. 3, 4, 5). Multiple samples also exhibited clear signs of vascular congestion (Figs. 3, 4, 5, 6, 7), as well as an inflammatory infiltration composed by lympho-histiocytic elements (Figs. 2, 3, 4, 5). Arterial structures exhibited the presence of a thickened media layer leading to diminished arterial patency, often to a level of sub-occlusion (Fig. 6). Furthermore, numerous nerve endings were observed between the altered muscle fibers, many showed visible damage accompanied by edema (Figs. 7, 8), fibrotic degeneration of the axons (Figs. 8, 9, 10) and thickening of the perineural sheath (Fig. 10). These histological changes were regularly seen independent of the age of the patient and the distance of the excision sites from the hernia border. Corresponding changes of the tissue structures were not detected in the control group of cadavers without hernia. In these cadavers, there was occasional evidence of an age-related interstitial fibrosis or a spotty fibroadipous dystrophy among the muscle fibers, while the nervous and vascular structures did not show any regressive modifications. No inflammatory infiltrate was detected in the biopsy specimens of the control group.

\section{Discussion}

The pathogenesis of inguinal hernia is poorly understood [6-9]. Furthermore, to our knowledge, there are only a few studies examining the histological modifications of the herniated groin [5, 10-12]. For example, the presence of fibrohyaline degeneration and inflammatory infiltration were reported in the muscular structure of the internal ring [5]. This study lead to the hypothesis that blockade of its

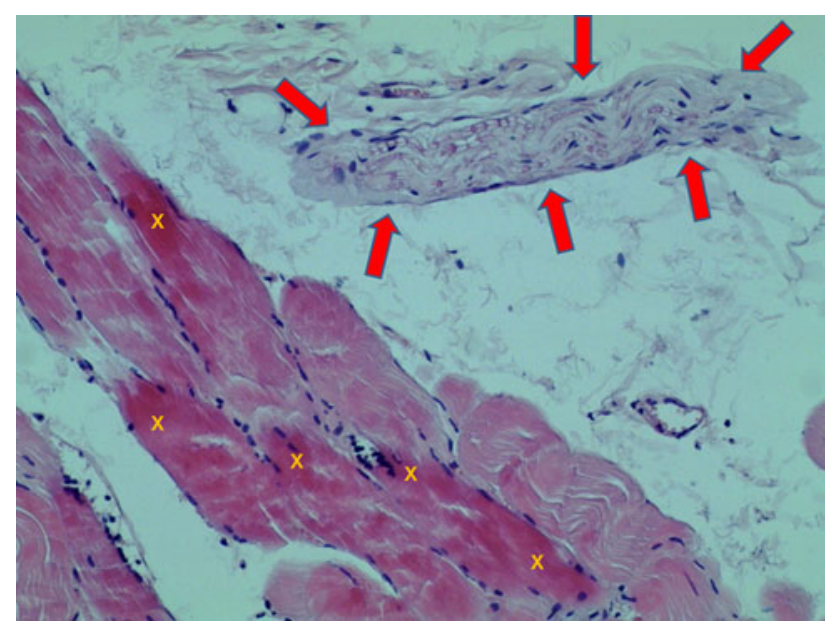

Fig. 2 Direct hernia in patient aged 47 years-tissue sample excised at $1 \mathrm{~cm}$ from hernia border: muscle bundle showing fibrotic (colored in pale red) and hyaline degeneration (marked with times symbol). Mild inflammatory infiltrate (black spotty elements). Nerve in longitudinal section with manifest edema (red arrows). PAS $\times 100$ (color figure online)

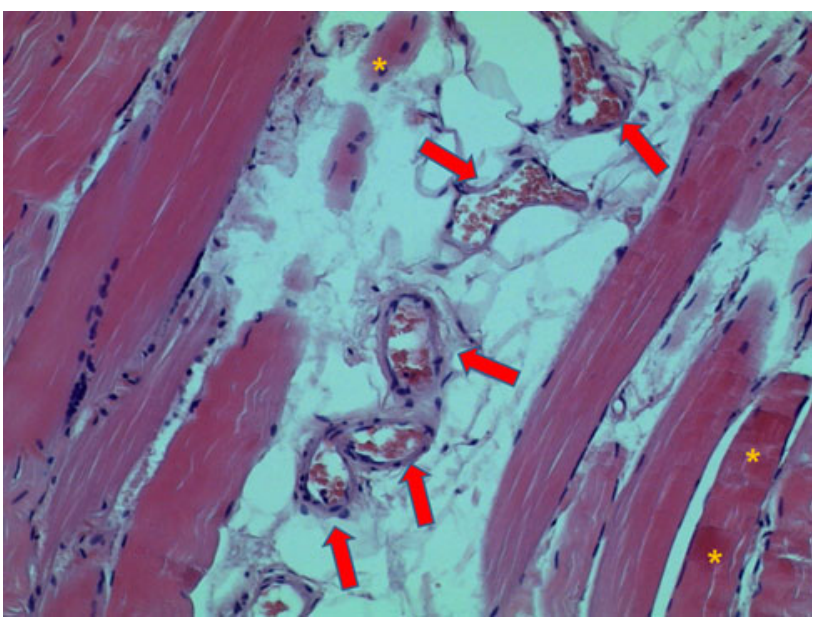

Fig. 3 Direct hernia in patient aged 74 years-tissue sample excised at $2 \mathrm{~cm}$ from hernia border: fibrotic (in pale red) and hyaline degeneration (marked with asterisk) of the muscle fibers. Fatty muscle dystrophy (white circular spots). Mild inflammatory infiltration (black spotty elements). Venous congestion (red arrows). PAS $\times 120$ (color figure online)

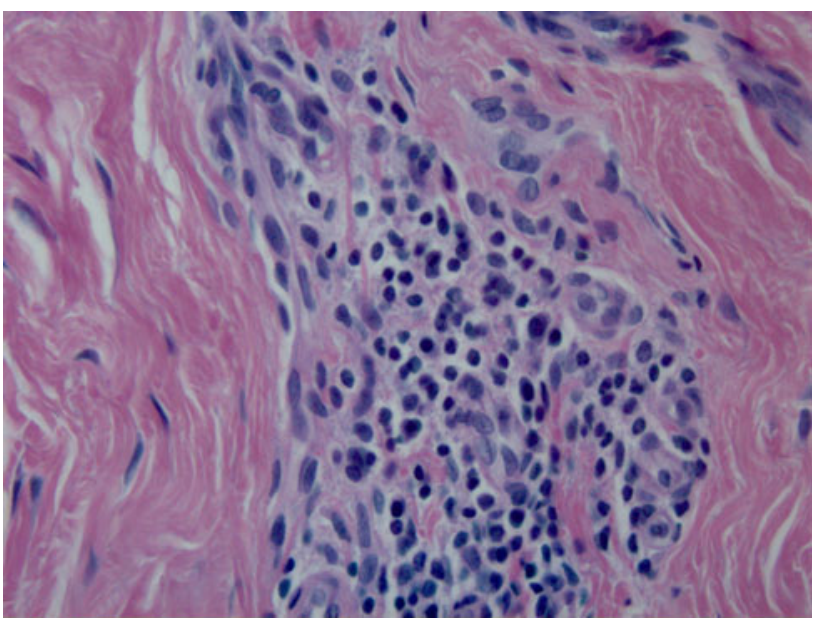

Fig. 4 Direct hernia in patient aged 56 years-tissue sample excised at $2 \mathrm{~cm}$ from hernia border: lymphocytic cluster in a surround of muscle fibrosis-hematoxylin eosin $\times 40$

shuttering action may be a potential factor associated with indirect hernia protrusion. However, this mechanism does not explain the occurrence of a direct inguinal hernia. In fact, despite the proximity between the internal ring and the fossa inguinalis media, the characteristics of these structures are crucially different, with the inguinal ring exhibiting a shuttering movement to create a dynamic hurdle, preventing visceral intrusion, while the myotendinous components of the fossa inguinalis media acting as a walllike barrier. Nevertheless, in some patients, this barrier becomes weakened until a direct hernia arises through it. In the present study, all 30 direct hernias from our patient 


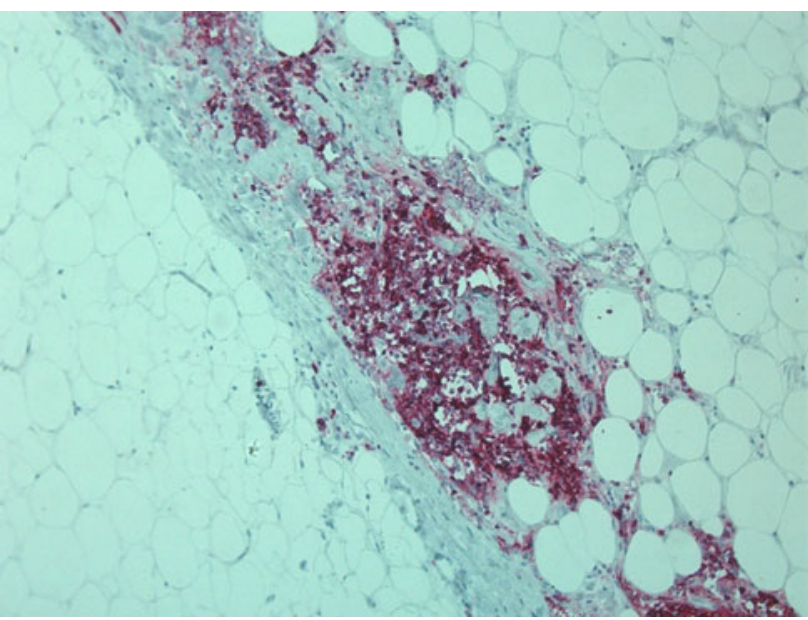

Fig. 5 Direct hernia in patient aged 68 years-tissue sample excised at $1 \mathrm{~cm}$ from hernia border: Lymphocytic cluster predominantly characterized by B phenotype (CD 20+) surrounded by fibroadipous dysplasia-CD $20 \times 20$

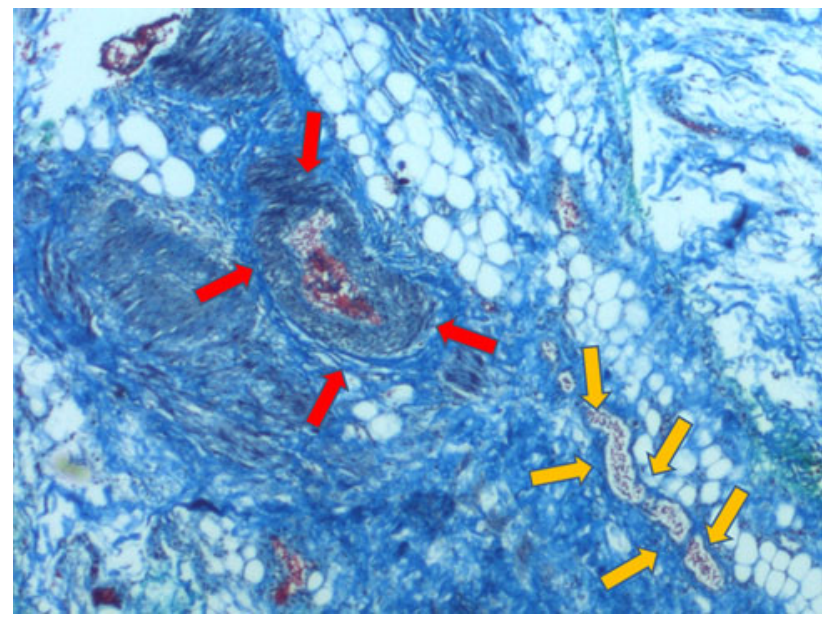

Fig. 6 Direct hernia in patient aged 64 years-tissue sample excised at $2 \mathrm{~cm}$ from hernia border: artery sub-occlusion due to thickened media (red arrow); venous congestion (yellow arrow) in a contest of severe fibroadipous dystrophy (white circular spots). Azan-Mallory $\times 2.5$ (color figure online)

sample demonstrated severe histological modifications with similar changes to those previously reported in indirect inguinal hernia [4], including inflammatory clusters composed of lympho-histiocytic elements within the muscle fibers. The presence of this inflammatory infiltrate, which exhibited the obvious characteristics of chronic inflammation, may be a result of steady compression of the groin structures as supported by injury of the neighboring vascular structures tend to support this thinking [13, 14]. Signs of acute inflammation including granulocytic elements were never detected in the tissue samples. The visible venous congestion leading to tissue edema is likely a consequence of chronic compression [13, 15]. This constant pressure

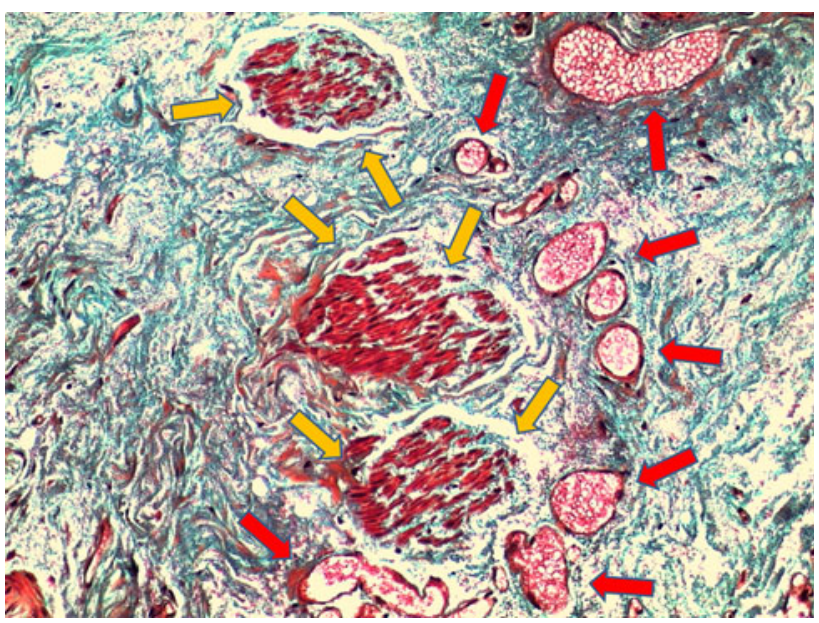

Fig. 7 Direct hernia in patient aged 42 years-tissue sample excised at $1 \mathrm{~cm}$ from hernia border: manifest venous congestion (red arrows), three nervous trunks with edema (yellow arrows). Hematoxylin eosin $\times 180$ (color figure online)

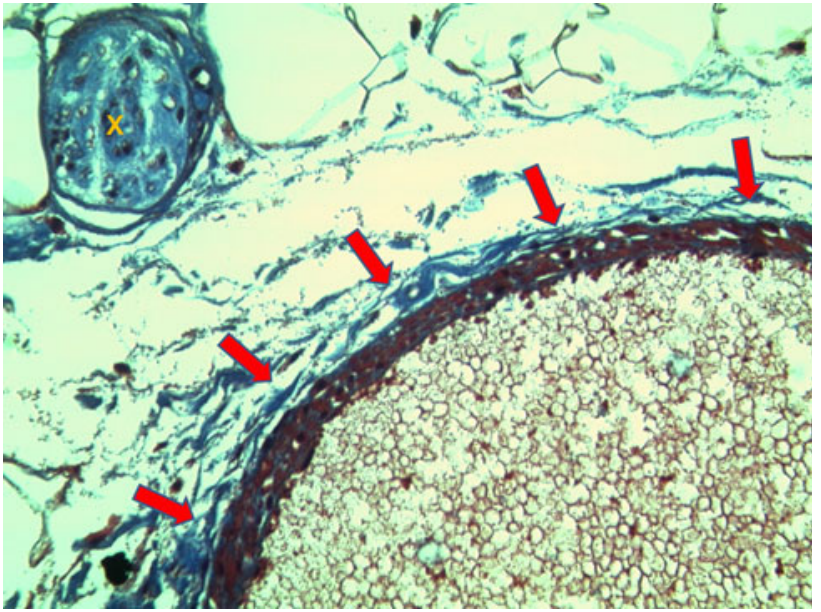

Fig. 8 Direct hernia in patient aged 69 years-tissue sample excised at $2 \mathrm{~cm}$ from hernia border: venous congestion (red arrows), nervous trunks with fibrotic degeneration and edema (marked with times symbol). Hematoxylin eosin $\times 180$ (color figure online)

injury would also explain the presence of medial hyperplasia leading to the detected sub-occlusion of the arteries. Both types of vascular damage lead to tissue damage including impaired tissue metabolism as a consequence of venous congestion and edema, diminished blood perfusion and chronic ischemia related to artery sub-occlusion.

Nervous structures were frequently identified in our specimens, and the nerve endings often demonstrated signs of injury due to edema, fibrotic degeneration and atrophy of the axons. Nearly all the altered nerve endings showed a manifest thickening of the perineural sheath, which is consistent with nerve damage in response to chronic compression of the nerve fiber $[16,17]$. The degeneration and atrophy of the nerve fibers likely leads to muscular 


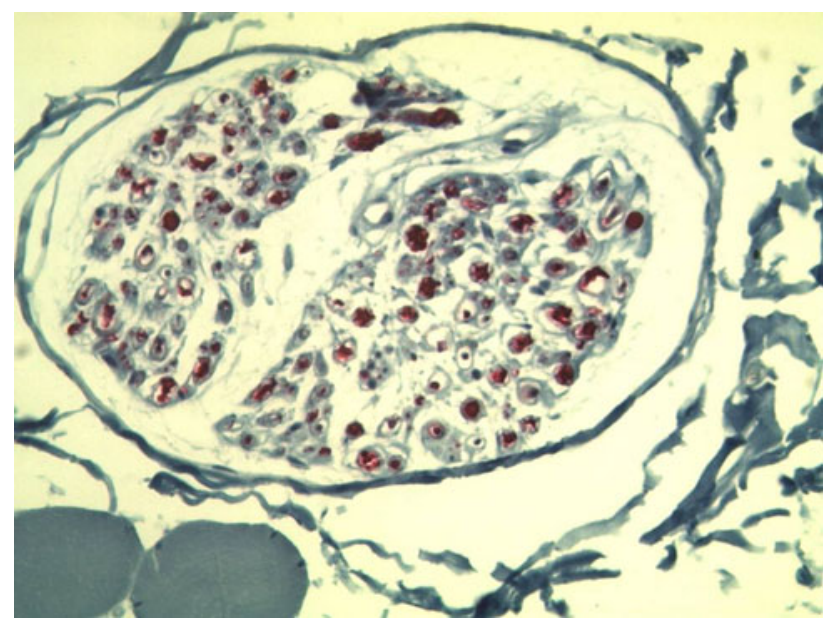

Fig. 9 Direct hernia in patient aged 52 years-tissue sample excised at $1 \mathrm{~cm}$ from hernia border: nerve degeneration due to fibrosis-NSE $\times 140$

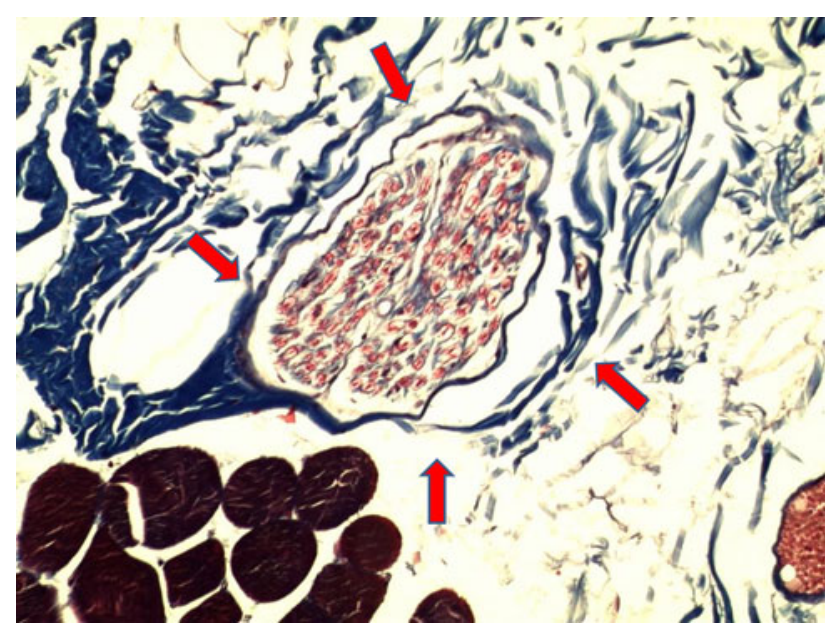

Fig. 10 Direct hernia in patient aged 45 years-tissue sample excised at $1 \mathrm{~cm}$ from hernia border: massive fibrotic degeneration of the nerve with thickening of the perineural sheet (red arrows)Masson $\times 100$ (color figure online)

dysfunction, and breakdown of motor nerve function may be a cause of atrophy in the muscle fibers. As a result, an additional structural and functional weakening of the muscular structure may exist. Such a relationship between reduced nervous impulse and histological changes of the muscles may be causative in the structural changes in the Hesselbach's triangle leading to decreased thickness of the muscular barrier. This may be precursor to direct hernia protrusion. The histological alterations observed in the present study may explain why the muscular structure of the groin is most severely damaged. We observed all degrees of muscular tissue degeneration in our tissue specimens, including hyaline, fibrotic and fatty dystrophy. Furthermore, major alterations of the muscular structure such as fibroadipous dystrophy of the muscle cells were constantly detected. The fatty substitution of the muscular shape caused an unusual subversion of the muscle cell. This regressive aspect of the muscular structure embodies a wellknown degeneration of the myocytes due to hypoxia, which is regularly reported in autopsied cardiac specimens as an evolution of myocardial infarction [18]. The biochemical steps leading from hypoxic changes until the fatty substitution of involve a long-term ischemia of the muscle bundles which eventually lose the contractile properties $[18,19]$. This results in fatty degeneration and to extreme weakening of the muscle (in this case the groin muscles). These data may explain the disbanding of the myotendinous barrier outlining the fossa inguinalis media and the resulting protrusion of a direct hernia. A sequence of images highlighting the macroscopic aspects of the fatty degeneration of the fossa inguinalis media is shown in Fig. 11. These pictures, taken during the indirect inguinal hernia repair procedure, reveal an evident fatty dystrophy of the fossa in the absence of hernia. A finger can easily penetrate the soft and fragile fossa inguinalis, opening a pathway for a direct hernia. Although there are limited reports of muscular damage following long-acting compression [20], our data suggest that muscle injuries are a consequence of a combined effect involving direct damage following the chronic compression exerted upon the muscle bundles, and indirect damage caused by chronic compression of the vascular structures and nerves of the groin, resulting in weakening of the muscular structures of the fossa inguinalis media.

As all the histological changes observed in our study can be caused by compressive damage, we suggest that chronic visceral compression leads to the weakening of the inguinal region through reduced physiological defense mechanisms. The steady formation of the hernia in a localized zone in an already weakened wall will exacerbate this protrusion process. If the compression injuries were an "effect" of the hernia being pushed through the hernia opening, then the impact of the compression would be highly localized to within a few $\mathrm{mm}$ of the hernia (epicenter). By a contrast, we found the same compressive impact at distances of up to $2 \mathrm{~cm}$ away from the hernia border, which is unlikely to have been caused by the hernia itself.

Furthermore, we found that damage was not diminished, which would be expected as you move out from the epicenter, but rather the even amount of compressive impact at all the biopsy sites suggests that the compression is coming from the abdominal side downwards-not from the hernia outwards.

\section{Conclusion}

We observed marked histological changes in the tissue at the border of direct inguinal hernia that were, in our opinion, 


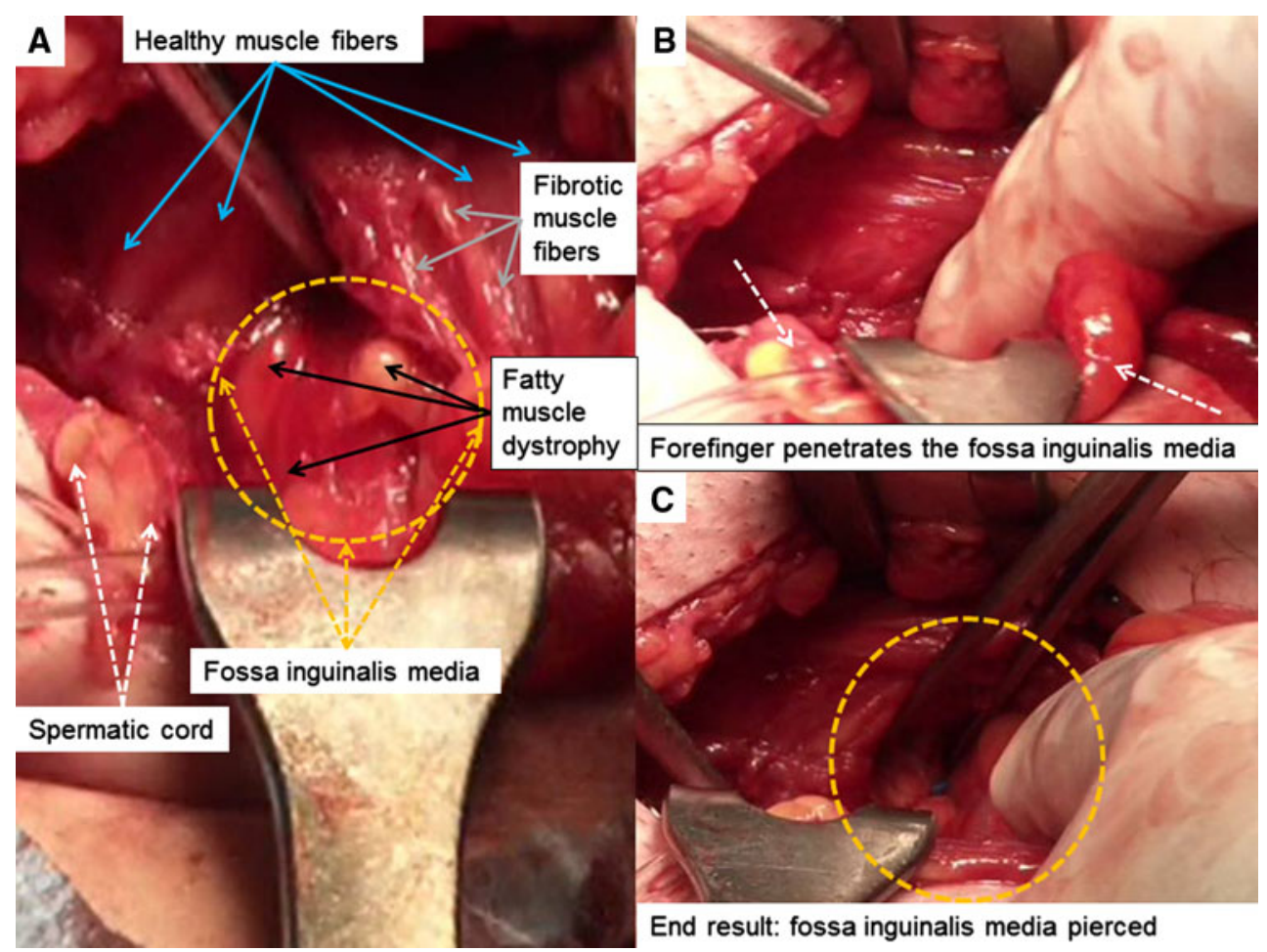

Fig. 11 Macroscopic representation of fatty degeneration of the fossa inguinalis media (yellow circle) during indirect inguinal hernia repair (white arrows: spermatic cord). a The whole fossa inguinalis shows manifest fatty dystrophy (black arrows). At this stage, no hernia can be recognized. Fibrotic altered muscle fibers are evident close to the fossa (gray arrows). The residual muscular structure has a normal aspect (blue arrows). b The forefinger penetrates across the fossa

indicative of chronic compressive damage exerted by the abdominal content against the lower abdominal wall. These changes provide strong evidence that direct inguinal hernia is a degenerative disease involving progressive weakening of the myotendinous components of the fossa inguinalis media, resulting in the protrusion of a direct hernia. These findings will be useful in developing new therapeutic strategies and defining advanced materials for hernia repair. Because of the degenerative nature of the disease, hernia repair should be based upon inducing an enhanced biological repair response and inguinal tissue regeneration.

Acknowledgments We thank Prof. Furio Silvestri and Prof. Rossana Bussani of the Anatomopathological Department of the University of Trieste (Italy) for the collaboration in purchasing the male cadavers without hernia for the control biopsy samples.

\section{References}

1. Friedman DW, Friedman DW, Boyd CD, Greco RS, Boyarsky AH, Mackenzie JW, Deak SB (1993) Increases in type III collagen gene expression and protein synthesis in patients with inguinal hernias. Ann Surg 218(6):754-760 inguinalis media without hinders (the white arrows indicate the spermatic cord). c The barrier composed by the fossa inguinalis (yellow circle) has been breached by simply finger piercing. The fascia transversalis is clearly detectable at the bottom. Thanks to the weakened barrier, the pathway for a forthcoming direct hernia has been achieved (color figure online)

2. Klinge U, Binnebösel M, Mertens PR (2006) Are collagens the culprits in the development of incisional and inguinal hernia disease? Hernia 10(6):472-477

3. Pans A (2002) Biochemical study of collagen in adult groin hernias. J Surg Res 95(2):107-113

4. Amato G, Sciacchitano T, Bell SG, Romano G, Cocchiara G, Lo Monte AI, Romano M (2009) Sphincter-like motion following mechanical dilation of the internal inguinal ring during indirect inguinal hernia procedure. Hernia 13:67-72

5. Amato G, Marasa L, Sciacchitano T, Bell SG, Romano G, Gioviale MC, Lo Monte AI, Romano M (2009) Histological findings of the internal inguinal ring in patients having indirect inguinal hernia. Hernia 13:259-262

6. Lytle WJ (1961) Anatomy and function in hernia repair. Proc R Soc Med 54:967-970

7. Flament JB (2006) Funktionelle Anatomie der Bauchwand. Chirurg 77:401-407

8. Stoppa R (1984-2004) Como se forma una hernia inguinal? Actualizacion en chirugia del aparato digestivo. 8:469-473; Fundacion MMA

9. Read RC (2003) Recent advances in the repair of groin herniation. Curr Probl Surg 40(1):13-79

10. Amato G, Ober E, Romano G, Salamone G, Agrusa A, Gulotta G, Bussani R (2011) Nerve degeneration in inguinal hernia specimens. Hernia 15:53-58

11. Amato G, Romano G, Salamone G, Agrusa A, Saladino VA, Silvestri F, Bussani R (2011) Damage to the vascular structures in inguinal hernia specimens. Hernia [Epub ahead of print] 
12. Amato G, Agrusa A, Romano G, Salamone G, Gulotta G, Silvestri F, Bussani R (2011) Muscle de generation in inguinal hernia specimens Hernia [Epub ahead of print]

13. Tozzi P (2007) The physiology of blood flow and artery wall. Springer editor, Sutureless Anastomoses, Darmstadt, Steinkopff, pp 12-24

14. Boyd GW (1980) The patho-physiology of chronic arterial hypertension: a hypothesis. Clin Exp Pharmacol Physiol 7(5):541-544

15. Kumar V, Abbas AK, Fausto N (2005) Blood vessel. In: Robbins SL, Cotran RS (eds) Pathologic basis of disease. Philadelphia, Saunders, pp 513-515

16. Kincaid JC, Stewart JD (1999) Focal peripheral neuropathies. J Clin Neuromuscul Dis 1(2):113
17. Penkert G (1998) Nerve compression syndrome-1. Chirurg 69:1114-1122

18. Silvestri F, Bussani R (1990) Hypoxic right ventricular cardiomyopathy. A morphological and pathogenetic study on the myocardial atrophy and fatty infiltration. Pathologica 82(1082): 593-616

19. Basso C, Thiene G, Corrado D, Angelini A, Nava A, Valente M (1996) Arrhythmogenic right ventricular cardiomyopathy. Dysplasia, dystrophy, or myocarditis? Circulation 94(5):983-991

20. Bai YH, Takemitsu M, Atsuta Y, Takemitsu Y (1998) Pathology study of rabbit calf muscles after repeated compression. J Orthop Sci 3(4):209-215 